
\title{
28 Research Suare \\ Prevalence of Ligamentous Knee Injuries in Pedestrian Versus Motor Vehicle Accidents
}

\section{Raymond Garrett Steinmetz}

University of Oklahoma Health Sciences Center

\section{Matthew McDonald}

University of Oklahoma Health Sciences Center

Shaun Tkach ( $\nabla$ shaun-tkach@ouhsc.edu )

University of Oklahoma Health Sciences Center

John Hamilton

University of Oklahoma Health Sciences Center

\section{Gregory Heigle}

University of Oklahoma Health Sciences Center

\section{Kimberly Hollabaugh}

University of Oklahoma Health Sciences Center

\section{David Teague}

University of Oklahoma Health Sciences Center

\section{Douglas Rowles}

University of Oklahoma Health Sciences Center

https://orcid.org/0000-0002-1168-4925

\section{Research article}

\section{Keywords:}

Posted Date: March 12th, 2020

DOI: https://doi.org/10.21203/rs.3.rs-16953/v1

License: (c) (1) This work is licensed under a Creative Commons Attribution 4.0 International License. Read Full License

Version of Record: A version of this preprint was published at BMC Musculoskeletal Disorders on June 10th, 2020. See the published version at https://doi.org/10.1186/s12891-020-03397-w. 


\section{Abstract}

Purpose: The purpose of this study was to determine the prevalence and characteristics of ligamentous knee injuries and to compare patient demographics, associated injuries and hospital stay to pedestrians who did not sustain a ligamentous knee injury.

Methods: A retrospective review of all adult patients presenting as pedestrians struck by a motor vehicle to a level 1 trauma center over a three-year period was performed. Demographics, length of stay, orthopedic and non-orthopedic traumatic injuries were recorded. Magnetic resonance imaging was reviewed for ligamentous, bony and chondral injuries.

Results: 539 patients were included. 67 (12.4\%) patients sustained a ligamentous knee injury and the majority of these were multi-ligamentous in nature. Patients with ligamentous knee injury were more likely to sustain traumatic brain injury, solid organ injury, cervical and lumbar spine injury, pelvic ring injuries, distal femur fractures, patella fractures, knee dislocations, tibial plateau fractures, tibial pilon fractures, and deep vein thrombosis when compared to patients who did not sustain ligamentous knee injury. Patients who sustained ligamentous knee injury were more likely to require hospital and intensive care admission and had a longer overall hospital stay.

Conclusion: Given the high prevalence of ligamentous knee injuries in this patient population, these patients should be thoroughly evaluated for a ligamentous knee injury. If ligamentous knee injury is suspected, MRI should be considered as a majority of these injuries involved multiple structures. Patients with ligamentous knee injuries often had multi-system injuries with resulting longer hospital stay when compared to those without ligamentous knee injuries.

\section{Introduction}

Pedestrian versus motor vehicle accidents are a common mechanism of injury in patients presenting to trauma facilities, especially in urban areas. Given the high energy associated with these injuries, the patients often have multiple systems injured, which leads to substantial morbidity and mortality [1]. Orthopedic injuries are very common and as shown by Brainard et al, lower extremity injuries are the most common orthopedic injuries in patients presenting as pedestrian versus motor vehicle accidents [2]. Despite this, not much is known about the prevalence of ligamentous knee injuries in this patient population.

The purpose of this study is to determine the prevalence and characteristics of ligamentous knee injuries in patients presenting as pedestrian versus motor vehicle accidents. We also sought to compare patient demographics, associated orthopedic and non-orthopedic injuries, intensive care stay and overall hospital stay to patients who did not have ligamentous knee injuries.

\section{Materials And Methods}


Institutional Review Board approval was obtained for this retrospective study. All patients greater than 18 years of age involved in pedestrian accidents presenting to a single institution level 1 trauma center from 2013-2017 were queried through our institution's trauma database. Patients who died within 24 hours of admission or died prior to orthopedic examination were excluded. Charts were retrospectively reviewed for age at time of injury, gender, height, weight, insurance status, hospital stay, intensive care stay, discharge destination, chest trauma, solid organ injury, vascular injury, facial and skull fractures, traumatic brain injury, gastrointestinal injury, genitourinary injury, spine and spinal cord injury, associated upper and lower extremity injuries. Ligamentous knee injuries were diagnosed either with physical exam or magnetic resonance imaging (MRI). For patients with ligamentous knee injuries, charts were reviewed for magnetic resonance imaging, specific ligaments or ligamentous complexes injured, meniscal injury, nerve injury, arterial injury, knee dislocation, chondral injury, bone bruise and subchondral fracture. For this studies, ligaments included were the anterior cruciate ligament $(A C L)$, posterior cruciate ligament $(\mathrm{PCL})$, medial collateral ligament $(\mathrm{MCL})$ and lateral collateral ligament/posterior lateral corner complex (LCL/PLC). For this study, the LCL/PLC complex includes the lateral collateral ligament, popliteus, popliteofibular ligament, arcuate ligament, fabellofibular ligament, long head of biceps femoris tendon and iliotibial band.

Statistical Analysis

Demographic and clinical characteristics were compared among patients with a ligamentous knee injury and patients without the injury. Chi-square tests were used to compare proportions and a T-test was used to compare continuous data. Wilcoxon rank sum p-value was used for length of stay data. The incidence of identified injuries was calculated along with a $95 \%$ confidence interval.

\section{Results}

557 patients were identified in our review and 18 patients were excluded due to death within 24 hours or death prior to adequate orthopedic evaluation leaving 539 patients to be included in this study. Table 1 shows the demographics of all included patients. 67 patients sustained ligamentous knee injuries giving a prevalence of $12.4 \%$. Seventeen patients sustained bilateral ligamentous knee injuries, giving a total of eighty-four ligamentous knee injuries. There were six knee dislocations, three vascular injuries and three peroneal nerve injuries.

MRI was available for 55/84 injured knees, the most common ligaments injured were anterior cruciate ligament (47), medial collateral ligament (45), lateral collateral ligament/ posterior lateral corner ligamentous complex (44) and the posterior cruciate ligament (38). Additionally, on MRI there were 37 instances of bone bruises or subchondral impaction fractures involving either the femoral condyles or tibial plateaus, 47 meniscal injuries and 9 acute chondral injuries. Table 2 shows the most common patterns of ligamentous injuries.

Tables 3 and 4 shows associated non-orthopedic and orthopedic injuries of both patients who sustained ligamentous knee injuries and those who did not. Patients who sustained ligamentous knee injuries were 
significantly more likely to have associated traumatic brain injury, solid organ injury and deep vein thrombosis of the lower extremity. As far as associated orthopedic injuries, patients who had ligamentous knee injuries were more likely to have cervical spine injury, lumbar spine injury, pelvic ring injuries, acetabular fractures, distal femur fractures, patella fractures, tibial plateau fractures, tibial pilon fractures and knee dislocations when compared to those who did not have ligamentous knee injuries.

Table 1 and Table 5 shows hospital and intensive care stay characteristics. Patients with ligamentous knee injuries were more likely to be admitted to the hospital and to the intensive care unit and had significantly longer hospital stay compared to those who did not have ligamentous knee injuries.

\section{Discussion}

Pedestrian versus motor vehicle accidents represent a high-energy mechanism that is common in urban areas and is associated with substantial morbidity and mortality [1]. Peng et al reviewed 5,000 pedestrians injured over a three year period by accessing a county database [1]. Their review showed that most common injuries included musculoskeletal (34.3\%), head and neck (30.0\%), external (24.4\%), abdomen and pelvis (3.9\%), chest $(2.4 \%)$ and spine (1.8\%). Additionally, they showed that at the time of discharge, $78 \%$ of patients had a temporary disability, $4 \%$ had a permanent handicap, and only $16 \%$ were functioning at preadmission capacity [1]. In another large review of 4,435 patients, Reith et al compared pedestrian versus motor vehicle patients to patients presenting as motor vehicle occupants [7]. They showed that the pedestrian cohort was more severely injured and had a higher rate of head injuries, pelvis injuries and lower extremity injuries [7]. In our review, patients who sustained a ligamentous knee injury were more likely to sustain traumatic brain injury and solid organ injury. Additionally, cervical spine injury, lumbar spine injury, pelvic ring injuries, acetabular fractures, distal femur fractures, patella fractures, tibial plateau fractures, tibial pilon fractures and knee dislocations when more common when compared to those patients who did not have ligamentous knee injuries. Lower extremity DVT was more common in patients who sustained ligamentous knee injury as well. These co-existing injuries should be acknowledged by the treating physician and could complicate the management of the ligamentous knee injury.

Orthopedic injuries, especially of the lower extremity, are very common in the pedestrian versus motor vehicle accident population. In one of the largest reviews of orthopedic trauma in this population, Brainard et al showed 96 injuries of the lower extremities in 115 patients who presented with this mechanism of injury [2]. Despite this, there has been little literature which looks specifically at ligamentous knee injuries in the pedestrian versus motor vehicle patient population. Our review shows a prevalence of $12.4 \%$ for ligamentous knee injuries in the pedestrian versus motor vehicle population. Becker et al reviewed 100 ligamentous knee injuries that presented to a level 1 trauma center, and found 23 patients (23\%) presented as pedestrians struck by a motor vehicle [3]. Moatshe et al evaluated 303 patients with a knee dislocation and showed that $5.9 \%$ of patients were pedestrians struck by a motor vehicle [4]. Ferry et al reviewed all patients who had a knee injury over a 14 year period and found that $14 \%$ of these patients were pedestrians who were struck by motor vehicles [5]. Forward et al showed that 
nearly half of the patients in their cohort sustained a knee injury following pedestrian versus motor vehicle accidents and $22 \%$ of those knee injuries were ligamentous in nature [6]. It can be difficult to diagnose a ligamentous knee injury in this patient population given that they often have injuries of multiple systems, thus a high index of suspicion is necessary. We recommend performing a thorough ligamentous knee exam in patients presenting with this mechanism. Not all patients in our review received $\mathrm{MRI}$, however, in those that did, the most common pattern of injury was $A C L+P C L+M C L+L C L / P L C$ complex and all but two were multi-ligamentous in nature. Additionally, we had a large number of concomitant intra-articular injuries which were identified on MRI. Given that a majority of these are multi-ligamentous, we recommend obtaining MRI in patients with suspected ligamentous knee injury who are stable enough to have the study and would benefit from potential repair or reconstruction.

Previous studies have reported on length of intensive care and hospital stay following pedestrian versus motor vehicle accidents. Peng et al showed that intensive care $6.5 \pm 0.3$ days and overall hospital stay was $7.4 \pm 0.2$ days [1]. In their comparison to patients who were occupants of motor vehicle accidents, Reith et al showed that pedestrians had a similar length of intensive care and overall hospital stay [7]. McElroy et al showed that pedestrian versus motor vehicle victims had an average hospital stay of 10.8 days and intensive care stay of $6 \pm 7.5$ days [8]. Specifically looking at knee injuries, Georgiadis et al showed that the average length of hospital stay in trauma patients who sustained a knee dislocation was 11.4 days [9]. Woodmass et showed that the average ICU stay in patients who sustained multiligamentous knee injuries was 2.8 days [10]. Additionally, they showed that $48 \%$ of patients with multiligamentous knee injuries required intensive care stay [10]. In our review, pedestrians who sustained a ligamentous knee injury had a longer overall hospital stay and were more likely to need inpatient admission and intensive care stay when compared to those who did not sustain a ligamentous knee injury.

This study does have limitations which need mentioned. This is a retrospective study and thus subject to selection bias. For this review the clinical follow up was very poor, therefore there could have been patients who were lost to follow up which sustained a ligamentous knee injury that was missed while inpatient. Additionally, patients were excluded if they died within 24 hours or died prior to orthopedic evaluation. These patients were often severely injured and potentially could have had ligamentous knee injuries as well. Given the poor follow up, we chose not to investigate how these patients fared after surgical or non-surgical management, thus this is a practice gap which could be evaluated with future studies. MRI was not obtained in all patients who sustained a ligamentous knee injury, typically this was due to patients who were clinically too unstable or if the knee injury was managed conservatively by the attending surgeons.

\section{Conclusion}

In conclusion, patients presenting as pedestrian versus motor vehicle have a high prevalence of ligamentous knee injuries and thus a high index of suspicion is necessary when evaluating these 
patients. Pedestrian versus motor vehicle accidents with ligamentous knee injuries were more likely to have multiple orthopedic and non-orthopedic injuries when compared to those without ligamentous knee injuries, thus representing the high energy of this mechanism. Patients sustaining ligamentous knee injuries were also more likely to be admitted to the hospital and intensive care unit and the length of overall hospital stay were also longer when compared to those who did not sustain ligamentous knee injury.

\section{Declarations}

Ethics Approval and Consent to Participate: Not Applicable

Consent for Publication: Not Applicable

Availability of Data and Materials: The datasets during and/or analyzed during the current study available from the corresponding author on reasonable request.

Competing Interests: The authors declare that they have no competing interests

Funding: No funding was obtained for this research

Authors Contributions:

RS made substantial contributions to the conception and design work of the research, the acquisition and analysis of data, the interpretation of data, and drafted the work.

MM made substantial contributions to the conception and design work of the research and the acquisition of data.

ST made substantial contributions in the acquisition and analysis of data and substantively revised the drafted manuscript.

$\mathrm{JH}$ made substantial contributions to the conception and design work of the research and the acquisition of data.

GH made substantial contributions in the acquisition and analysis of data.

$\mathrm{KH}$ made substantial contributions to the interpretation of data.

DT acted as a faculty sponsor as well as provided feedback and direction during the research process

DR acted as a faculty sponsor as well as provided feedback and direction during the research process Acknowledgements: Not Applicable

\section{References}


1. Peng, R. Y., \& Bongard, F. S. (1999). Pedestrian versus motor vehicle accidents: an analysis of 5,000 patients. Journal of the American College of Surgeons, 189(4), 343-348. doi: 10.1016/s10727515(99)00166-0

2. Brainard, B. J., Slauterbeck, J., \& Benjamin, J. B. (1992). Fracture Patterns and Mechanisms in Pedestrian Motor-Vehicle Trauma. Journal of Orthopaedic Trauma, 6(3), 279-282. doi: 10.1097/00005131-199209000-00002

3. Becker, E. H., Watson, J. D., \& Dreese, J. C. (2013). Investigation of Multiligamentous Knee Injury Patterns With Associated Injuries Presenting at a Level I Trauma Center. Journal of Orthopaedic Trauma, 27(4), 226-231. doi: 10.1097/bot.0b013e318270def4

4. Moatshe, B. G., Dornan, G., Løken, S., Ludvigsen, T. C., Laprade, R. F., \& Engebretsen, L. (2017). Knee Dislocations Demographics and Associated Injuries: A Prospective Review of 303 Patients. Arthroscopy: The Journal of Arthroscopic \& Related Surgery, 33(10). doi: 10.1016/j.arthro.2017.08.195

5. Ferry, T., Bergström, U., Hedström, E. M., Lorentzon, R., \& Zeisig, E. (2013). Epidemiology of acute knee injuries seen at the Emergency Department at Umeå University Hospital, Sweden, during 15 years. Knee Surgery, Sports Traumatology, Arthroscopy, 22(5), 1149-1155. doi: 10.1007/s00167013-2555-3

6. Forward, D. P., Coffey, F., Wallace, W. A., \& Ellis, J. (2003). Knee injuries sustained in pedestrian road traffic accidents. Orthopaedic Proceedings, 85-B(supp), 13-13.

7. Reith, G., Lefering, R., Wafaisade, A., Hensel, K. O., Paffrath, T., Bouillon, B., \& Probst, C. (2015). Injury pattern, outcome and characteristics of severely injured pedestrian. Scandinavian Journal of Trauma, Resuscitation and Emergency Medicine, 23(1). doi: 10.1186/s13049-015-0137-8

8. McElroy, L. M., Juern, J. J., Bertleson, A., Xiang, Q., Szabo, A., \& Weigelt, J. (2013). A single urban center experience with adult pedestrians struck by motor vehicles. WMJ, 112(3), 117-122.

9. Georgiadis, A. G., Mohammad, F. H., Mizerik, K. T., Nypaver, T. J., \& Shepard, A.D. (2012). Changing Presentation of Knee Dislocation and Vascular Injury: From High-Energy Trauma to Low-Energy Falls in the Morbidly Obese. Journal of Vascular Surgery, 56(4), 1189. doi: 10.1016/j.jvs.2012.08.018

10. Woodmass, J. M., Johnson, N. R., Mohan, R., Krych, A. J., Levy, B. A., \& Stuart, M. J. (2017). Polytraumatic multi-ligament knee injuries: is the knee the limiting factor? Knee Surgery, Sports Traumatology, Arthroscopy, 26(9), 2865-2871. doi: 10.1007/s00167-017-4784-3

\section{Tables}

Due to technical limitations, Tables 1 - 5 are only available for download from the Supplementary Files section.

\section{Supplementary Files}

This is a list of supplementary files associated with this preprint. Click to download. 
- Table2.docx

- Table1.docx

- Table4.docx

- Table5.docx

- Table3.docx 